\title{
Myths Associated with Menarche and Menstruation among Tribal Females of Kargil District, Jammu and Kashmir
}

\author{
Sabiya Asmat ${ }^{*}$, Sarika Manhas ${ }^{2}$ and Tashi Dolkar ${ }^{3}$ \\ ${ }^{1}$ Krishi Vigyan Kendra, Kargil I, S. K University of Agriculture Science \& Technology of \\ Kashmir, Jammu and Kashmir, India \\ ${ }^{2}$ Department of Home Science, University of Jammu, Jammu and Kashmir, India \\ ${ }^{3}$ Krishi Vigyan Kendra, Kargil II, S. K University of Agriculture Science \& Technology of \\ Kashmir, Jammu and Kashmir, India \\ *Corresponding author
}

\section{A B S T R A C T}

Despite being a phenomenon unique to girls, this has always been surrounded by secrecy and myths in many societies. Taboos surrounding menstruation exclude women and girls

\section{Keywords}

Menstruation,

Tribals, Kargil,

Myths and

restrictions

Article Info

Accepted:

18 May 2019

Available Online:

10 June 2019 from many aspects of social and cultural life. Some of these are helpful, but others have potentially harmful implications (Kaiser, 2008). The main objective of the present study was to assess the myths associated with menarche and menstruation among tribal females of Kargil. The sample for the study comprised of 520 tribal respondents of Kargil district, which comprised of 320 unmarried adolescent girls in the age group of 14-18 years, 100 mothers and 100 grandmothers. Interaction with the females of the two tribes highlight that there are many myths and restrictions associated with menarche and menstruation. These myths and restrictions have generally been passed from older generation to newer generation for many years now. It is in fact, difficult to cite when and from where these myths and restrictions came into existence, but majority of menstruating females emphasized that they agreed with these prevailing myths. The older generation of females especially the grandmothers and mothers followed and believed in these myths. However, adolescent girls either thought of these myths as erroneous or were not sure about the authenticity or practical viability of these myths.

\section{Introduction}

Menstruation has been surrounded by taboos and myths that eliminate women from many aspects of socio-cultural life. Many cultures hold on different beliefs and myths that restrict women from daily activities and hygienic health practices, which lead to adverse outcomes such as infection (Dasgupta and Sarkar, 2008). Menstrual cycle is an integral part of a female's life which due to the superstitions and myths followed by the families and others in the community, gets dangerously ignored and is not given importance. Many communities world over have gone through centuries of myths and 
taboos that have created an ideology of the female body which has continued to impact the lives of women and girls in modern society. Religious teachings, traditional practices and cultural beliefs, have all played their role in the ideology of stigmatizing the menstruating woman. Sadly such myths have led to $48 \%$ of girls in Iran, $10 \%$ in India, and $7 \%$ in Afghanistan believing that menstruation is a disease, it is the removal of bad blood from the body, rather than a natural and healthy part of adolescence or young adulthood (SOS Children's Village, 2014). In rural Nepal, menstruating girls are sometimes forced out of their homes and forbidden from contact with people, animals and even plants. The practice of sequestering girls in menstrual huts is harmful to them, and sometimes fatal (Dineen, 2019).

Our menstrual practices are embedded strongly in religious and cultural contexts (Shuttle and Redgrove, 2005) and have in fact become a part of our daily life. The rural and tribal adolescent girls are especially vulnerable on account of limited access to health care and a dominant socio-cultural ecology (Singh, 2006; Khanna et al., 2005). With this as background, the present study explores the Myths associated with Menarche and Menstruation among Tribals of Kargil Jammu and Kashmir state of India.

\section{Materials and Methods}

\section{Sample and sample size}

The sample for the study was divided into 3 groups

\section{Group I}

Comprised of 320 unmarried adolescent girls, in the age group of 14-18 years, selected equally from Balti tribe and Purig tribe, of Kargil District.

\section{Group II}

Consisted of 100 mothers (50 from Purig tribe and 50 from Balti tribe), who had at least one adolescent daughter, who were again selected randomly and equally, from the two tribes.

\section{Group III}

Consisted of 100 grandmothers (50 from Purig tribe and 50 from Balti tribe), who had at least one adolescent granddaughter experiencing menstruation, drawn randomly from Kargil block and Sankoo block of Kargil District. A self-structured interview schedule, Checklist and focus group discussion was used, to assess the information of sample, females regarding menarche and menstruation

\section{Results and Discussion}

\section{Myth: "Menstruating Women and Girls} are Unclean"

Fact: Menstruation is a sign of health and normal development. Menstrual blood is the same as blood from anywhere else in the body and is usually sterile. Girls should always maintain good hygiene throughout their menstrual periods

Data in table 1 shows that Majority of females across generations (girls $86.2 \%$, mothers $96 \%$ and grandmothers $100 \%$ ) accepted the myth that, menstruating women and girls are unclean. These respondents reported that because of its impurity the menstruating women were not supposed to offer prayers during menstruation. They further added that we do not know why it is considered as impure but it directed by our God "Allah" and being a follower of Islam we have to believe this as true. The myth that menstruating women are unclean and polluting is widespread in the cultures of many developing countries. It includes the myth that 
women's excretions are considered to be polluting during menstruation (and childbirth as well) (Kumar and Srivastava, 2011; Yesmin et al., 2008; Ten, 2007). In Mali, some tribes have exclusion huts, where women are sent to stay during their menstruation, excommunicating them from their families for 4-5 days, however they are not excluded from work (Kivuvani, 2013). Statistical analysis show highly significant differences across generations and also between girls of Purig and Balti tribes. No doubt that majority of respondents viewed this statement as true but there are few respondents ( $9.3 \%$ girls and $4 \%$ mothers) who thought that this wasn't true. Comparatively more Balti girls agreed with this than the Purig girls. However, no significant differences were seen between mothers and grandmothers of these two tribes, as both held the similar views.

Myth: Menstrual Blood is "Dirty Blood" that Does not Come out of the Body when one misses her Period

Fact: Blood discharged during periods is regular blood in the body. It is also not impure. Menstruation is a sign of health and normal development. Menstrual blood is the same as blood from anywhere else in the body and is usually sterile. Girls should always maintain good hygiene throughout their menstrual periods.

Data contained in the table 2 depicts that majority $(68.1 \%$ girls, $69 \%$ mothers and $100 \%$ grandmothers) of respondents reported that it is true that menstrual blood is "dirty blood" that does not come out of the body when one misses her period. These respondents also reported that monthly cycle is important for every menstruating woman, to flush out the toxins and impure blood from the body as it helps to clean the body. A woman tends to have clear and glowing skin after finishing her monthly cycle. Data indicates that just like other parts of the world, here also menstrual blood is considered as 'dirty'. George (2014) found that menstruation is dirty, and a menstruating girl is a powerful, polluting thing, a thing to be feared and shunned. Similarly, most of the Kargil women also viewed menstrual blood as dirty, however the reasons are different. Findings also show that this myth is popular mostly among older generation compared to younger generation. This indicates that younger girls may be having more relevant information, probably from schools books (science books) and media. Statistical analysis shows that significant difference exist among the three generations and between early and late adolescent girls $(\mathrm{p}=0.00)$ as more late adolescent girls viewed this as true than early adolescent girls. Tribe wise analysis show that more Balti adolescent girls and mothers were likely to think of this statement as true than Purig adolescent girls and mothers (highly significant difference was found between them, $\mathrm{p}=0.00$ ). But no difference was found between Balti grandmothers and Purig grandmothers as all the sample grandmothers agreed that menstrual blood is "dirty blood" that does not come out of the body when one misses her period.

Myth: "Menstruation Symbolizes Psychological and Physical Maturity among Females"

Fact: a) A girl's body is still developing after she has started menstruating. Getting married and having a baby before the age of 18 can lead to health problems for the mother and child.

b) Menstruation is an important stage in a girl's maturation, yet it does not mean a girl is ready for marriage

The data in the figure 1 depicts that majority of the mothers $(76 \%)$ and grandmothers 
$(53 \%)$ of Kargil believed that "menstruation symbolises psychological and physical maturity among females". The older generation females mostly felt that, girls are ready of marriage after menarche. She is physically prepared to bear a child to continue the human race/family lineage. However, majority of adolescent girls (43.7\%) did not believe in this, as they did not see any direct linkage between puberty and maturity. Only $26.5 \%$ of the sample girls considered this as true, because they felt that the information passed from mothers and grandmothers can never be incorrect. Another $29.6 \%$ of these girls were not sure about the accuracy of this belief. These respondents reported that in olden days menarche was not considered as a sign of marriage as then many girls even got married at 10, 12 or 13 years, much before attaining menarche. Statistical analysis showed that there is a highly significant difference $(p=0.000)$ across the three generations on this belief.

Tribe wise, more Purig respondents in all the three age groups $(33.1 \%$ girls $100 \%$ and mothers and grandmothers) believed in this statement as compared to their Balti counter parts $(20 \%$ girls, 52\% mothers and 6\% grandmothers). Highly significant difference $(\mathrm{p}=0.000)$ was also found between early adolescent girls and late adolescent girls; as majority (43.2\%) of early adolescent girls weren't sure about this belief whereas, majority $(63.4 \%)$ of late adolescent girls reported that this was not true.

Table.1 Menstruating Women and Girls Are Unclean

\begin{tabular}{|c|c|c|c|c|c|c|c|c|c|c|c|c|c|c|c|}
\hline \multirow[b]{2}{*}{ Response } & \multicolumn{2}{|c|}{ Purig } & \multicolumn{2}{|c|}{ Balti } & \multirow{2}{*}{$\begin{array}{c}\text { Early } \\
\text { Ado. } \\
14-16 \\
\text { Years } \\
\text { N=164 }\end{array}$} & \multirow{2}{*}{$\begin{array}{c}\begin{array}{c}\text { Late } \\
\text { Ado }\end{array} \\
16-18 \\
\text { Years } \\
\text { N=156 }\end{array}$} & \multicolumn{2}{|c|}{ Girls } & \multicolumn{2}{|c|}{ Mothers } & \multicolumn{2}{|c|}{ Grandmothers } & \multicolumn{3}{|c|}{ Females } \\
\hline & $\begin{array}{l}14-16 \\
\text { Years } \\
N=77\end{array}$ & $\begin{array}{l}16-18 \\
\text { Years } \\
\mathrm{N}=83\end{array}$ & $\begin{array}{l}14-16 \\
\text { Years } \\
N=87\end{array}$ & $\begin{array}{l}16-18 \\
\text { Years } \\
N=73\end{array}$ & & & $\begin{array}{c}\text { Purig } \\
\mathrm{N}=16 \\
0\end{array}$ & $\begin{array}{c}\text { Balti } \\
\mathrm{N}=16 \\
0\end{array}$ & $\begin{array}{l}\text { Purig } \\
\mathrm{N}=50\end{array}$ & $\begin{array}{l}\text { Balti } \\
\mathbf{N}=\mathbf{5 0}\end{array}$ & $\begin{array}{l}\text { Purig } \\
\mathrm{N}=50\end{array}$ & $\begin{array}{l}\text { Balti } \\
\mathbf{N}=\mathbf{5 0}\end{array}$ & $\begin{array}{c}\text { Girls } \\
\mathrm{N}=32 \\
0\end{array}$ & $\begin{array}{l}\text { Mothers } \\
\mathbf{N}=100\end{array}$ & $\begin{array}{c}\text { Grand } \\
\text { mothers } \\
\mathrm{N}=100\end{array}$ \\
\hline True & $\begin{array}{c}73 \\
(94.8)\end{array}$ & $\begin{array}{c}45 \\
(58.4)\end{array}$ & $\begin{array}{c}87 \\
(100)\end{array}$ & $\begin{array}{c}71 \\
(97.2)\end{array}$ & $\begin{array}{c}160 \\
(100)\end{array}$ & $\begin{array}{c}116 \\
(74.3)\end{array}$ & $\begin{array}{c}118 \\
(73.7)\end{array}$ & $\begin{array}{c}158 \\
(98.7)\end{array}$ & $\begin{array}{c}46 \\
(92)\end{array}$ & $\begin{array}{c}50 \\
(100)\end{array}$ & $\begin{array}{c}50 \\
(100)\end{array}$ & $\begin{array}{c}50 \\
(100)\end{array}$ & $\begin{array}{c}276 \\
(86.2)\end{array}$ & $\begin{array}{c}96 \\
(96)\end{array}$ & $\begin{array}{c}100 \\
(100)\end{array}$ \\
\hline False & - & $\begin{array}{c}30 \\
(38.9)\end{array}$ & - & - & 0 & $\begin{array}{c}30 \\
(19.2)\end{array}$ & $\begin{array}{c}30 \\
(18.7)\end{array}$ & - & $\begin{array}{c}4 \\
(8)\end{array}$ & - & - & - & $\begin{array}{c}30 \\
(9.3)\end{array}$ & $\begin{array}{c}4 \\
(4)\end{array}$ & \\
\hline \multirow[t]{2}{*}{ Not sure } & $\begin{array}{c}4 \\
(5.1)\end{array}$ & $\begin{array}{c}8 \\
(9.6)\end{array}$ & - & $\begin{array}{c}2 \\
(2.7)\end{array}$ & $\begin{array}{c}4 \\
(2.4)\end{array}$ & $\begin{array}{c}10 \\
(6.4)\end{array}$ & $\begin{array}{c}12 \\
(7.5)\end{array}$ & $\begin{array}{c}2 \\
(1.2)\end{array}$ & - & - & - & - & $\begin{array}{c}14 \\
(4.3)\end{array}$ & - & \\
\hline & \multicolumn{6}{|c|}{$\begin{array}{l}\text { Chi-sq between early and late adolescent girls } 39.4^{* *} \text {, } \\
\qquad \mathrm{P}=0.0, \mathrm{df}=2\end{array}$} & \multicolumn{2}{|c|}{$\begin{array}{l}\text { Chi-sq between } \\
\text { Purig and Balti } \\
\text { girls } 42.9^{* *} \\
\mathrm{P}=0.0, \mathrm{df}=2\end{array}$} & \multicolumn{2}{|c|}{$\begin{array}{c}\text { Chi-sq between } \\
\text { Purig and Balti } \\
\text { mothers } 4.16 \text {, } \\
\mathrm{p}=0.041, \mathrm{df}=1\end{array}$} & & - & \multicolumn{3}{|c|}{$\begin{array}{c}\text { Chi-sq across generation } \\
=22.17 * *, \mathrm{p}=0.000 \\
\mathrm{df}=4\end{array}$} \\
\hline
\end{tabular}

Table.2 Menstrual Blood is "Dirty Blood" that does not come out of the Body when one misses her period

\begin{tabular}{|c|c|c|c|c|c|c|c|c|c|c|c|c|c|c|c|}
\hline \multirow[t]{2}{*}{ Responses } & \multicolumn{2}{|c|}{ Purig } & \multicolumn{2}{|c|}{ Balti } & \multirow{2}{*}{$\begin{array}{c}\text { Early } \\
\text { Ado. } \\
14-16 \\
\text { Years } \\
\mathrm{N}= \\
164\end{array}$} & \multirow{2}{*}{\begin{tabular}{|c|} 
Late \\
Ado \\
$16-18$ \\
Years \\
$\mathrm{N}=156$
\end{tabular}} & \multicolumn{2}{|c|}{ Girls } & \multicolumn{2}{|c|}{ Mothers } & \multicolumn{2}{|c|}{ Grandmothers } & \multicolumn{3}{|c|}{ Females } \\
\hline & $\begin{array}{l}14-16 \\
\text { Years } \\
N=77\end{array}$ & $\begin{array}{l}16-18 \\
\text { Years } \\
\mathrm{N}=83\end{array}$ & $\begin{array}{l}14-16 \\
\text { Years } \\
N=87\end{array}$ & $\begin{array}{l}16-18 \\
\text { Years } \\
N=73\end{array}$ & & & $\begin{array}{c}\text { Purig } \\
\mathrm{N}=16 \\
0\end{array}$ & $\begin{array}{c}\text { Balti } \\
\mathbf{N}=160\end{array}$ & $\begin{array}{l}\text { Purig } \\
\mathrm{N}=50\end{array}$ & $\begin{array}{l}\text { Balti } \\
\mathrm{N}=\mathbf{5 0}\end{array}$ & $\begin{array}{l}\text { Purig } \\
\mathrm{N}=\mathbf{5 0}\end{array}$ & $\begin{array}{l}\text { Balti } \\
\mathbf{N}=\mathbf{5 0}\end{array}$ & $\begin{array}{c}\text { Girls } \\
\mathbf{N}=32 \\
0\end{array}$ & $\begin{array}{c}\text { Moth } \\
\text { ers } \\
\mathbf{N}=10 \\
0\end{array}$ & $\begin{array}{c}\text { Grand } \\
\text { mother } \\
\text { s } \\
\mathrm{N}=100\end{array}$ \\
\hline True & $\begin{array}{c}44 \\
(57.1)\end{array}$ & $\begin{array}{c}52 \\
(67.5)\end{array}$ & $\begin{array}{c}57 \\
(65.5)\end{array}$ & $\begin{array}{c}65 \\
(89.0)\end{array}$ & $\begin{array}{c}101 \\
(61.5)\end{array}$ & $\begin{array}{l}117 \\
\text { (75) }\end{array}$ & $\begin{array}{c}96 \\
(60)\end{array}$ & $\begin{array}{c}122 \\
(76.25)\end{array}$ & $\begin{array}{c}19 \\
(38)\end{array}$ & $\begin{array}{c}50 \\
(100)\end{array}$ & $\begin{array}{c}50 \\
(100)\end{array}$ & $\begin{array}{c}50 \\
(100)\end{array}$ & $\begin{array}{c}218 \\
(68.1)\end{array}$ & $\begin{array}{l}69 \\
(69)\end{array}$ & $\begin{array}{c}100 \\
(100)\end{array}$ \\
\hline False & - & $\begin{array}{c}14 \\
(18.1)\end{array}$ & - & $\begin{array}{c}4 \\
(5.4)\end{array}$ & - & $\begin{array}{c}18 \\
(11.5)\end{array}$ & $\begin{array}{c}14 \\
(8.7)\end{array}$ & $\begin{array}{c}4 \\
(2.5)\end{array}$ & $\begin{array}{c}16 \\
(32)\end{array}$ & - & - & - & $\begin{array}{c}18 \\
(5.6)\end{array}$ & $\begin{array}{c}16 \\
(16)\end{array}$ & - \\
\hline \multirow[t]{2}{*}{ Not sure } & $\begin{array}{c}33 \\
(48.8)\end{array}$ & $\begin{array}{c}17 \\
(20.4)\end{array}$ & $\begin{array}{c}30 \\
(34.4)\end{array}$ & $\begin{array}{c}4 \\
(5.4)\end{array}$ & $\begin{array}{c}63 \\
(38.4)\end{array}$ & $\begin{array}{c}21 \\
(13.4)\end{array}$ & $\begin{array}{c}50 \\
(31.2)\end{array}$ & $\begin{array}{c}34 \\
(21.2)\end{array}$ & $\begin{array}{c}15 \\
(30)\end{array}$ & - & - & - & $\begin{array}{c}84 \\
(26.2)\end{array}$ & $\begin{array}{l}15 \\
(15)\end{array}$ & - \\
\hline & \multicolumn{2}{|c|}{ Chi-sq between } & $\begin{array}{r}\text { arly and } \\
\mathrm{P}=0.0\end{array}$ & $\begin{array}{l}\text { ite adole } \\
\mathrm{df}=1\end{array}$ & ent girls & $6.8 * *$ & \multicolumn{2}{|c|}{$\begin{array}{c}\text { Chi-sq between } \\
\text { Purig and Balti } \\
\text { girls tribe wise } \\
11.7 * *, \mathrm{P}=0.002, \\
\mathrm{df}=2\end{array}$} & \multicolumn{2}{|c|}{$\begin{array}{c}\text { Chi-sq } \\
\text { betweenPurig and } \\
\text { Balti mothers tribe } \\
\text { wise } 44.9^{* *} \\
\mathrm{p}=000, \mathrm{df}=2\end{array}$} & \multicolumn{2}{|c|}{-} & \multicolumn{3}{|c|}{$\begin{array}{l}\text { Chi-sq across generation } \\
=55.6^{* *}, \mathrm{p}=0.000, \mathrm{df}=4\end{array}$} \\
\hline
\end{tabular}


Table.3 It is very disgraceful for females when a man finds out that a woman is having her period

\begin{tabular}{|c|c|c|c|c|c|c|c|c|c|c|c|c|c|c|c|}
\hline \multirow[t]{2}{*}{ Response } & \multicolumn{2}{|l|}{ Purig } & \multicolumn{2}{|l|}{ Balti } & \multirow{2}{*}{$\begin{array}{c}\begin{array}{l}\text { Early } \\
\text { Ado. }\end{array} \\
14-16 \\
\text { Years } \\
\text { N=164 }\end{array}$} & \multirow{2}{*}{$\begin{array}{l}\begin{array}{l}\text { Late } \\
\text { Ado }\end{array} \\
16-18 \\
\text { Years } \\
\mathrm{N}=156\end{array}$} & \multicolumn{2}{|l|}{ Girls } & \multicolumn{2}{|c|}{ Mothers } & \multicolumn{2}{|c|}{ Grandmothers } & \multicolumn{3}{|c|}{ Females } \\
\hline & $\begin{array}{l}14-16 \\
\text { Years } \\
N=77\end{array}$ & $\begin{array}{l}16-18 \\
\text { Years } \\
\mathrm{N}=83\end{array}$ & $\begin{array}{l}14-16 \\
\text { Years } \\
N=87\end{array}$ & $\begin{array}{l}16-18 \\
\text { Years } \\
N=73\end{array}$ & & & $\begin{array}{c}\text { Purig } \\
\mathrm{N}=160\end{array}$ & $\begin{array}{c}\text { Balti } \\
N=160\end{array}$ & $\begin{array}{l}\text { Purig } \\
\mathrm{N}=50\end{array}$ & $\begin{array}{l}\text { Balti } \\
\mathbf{N}=\mathbf{5 0}\end{array}$ & $\begin{array}{l}\text { Purig } \\
\mathrm{N}=50\end{array}$ & $\begin{array}{l}\text { Balti } \\
\mathbf{N}=\mathbf{5 0}\end{array}$ & $\begin{array}{c}\text { Girls } \\
\mathbf{N}=\mathbf{3 2 0}\end{array}$ & $\begin{array}{c}\text { Mothers } \\
\mathbf{N}=100\end{array}$ & $\begin{array}{c}\text { Grand } \\
\text { mothers } \\
N=100\end{array}$ \\
\hline True & $\begin{array}{c}77 \\
(100)\end{array}$ & $\begin{array}{c}83 \\
(100)\end{array}$ & $\begin{array}{c}87 \\
(100)\end{array}$ & $\begin{array}{c}73 \\
(100)\end{array}$ & $\begin{array}{c}164 \\
(100)\end{array}$ & $\begin{array}{c}156 \\
(100)\end{array}$ & $\begin{array}{c}160 \\
(100)\end{array}$ & $\begin{array}{c}160 \\
(100)\end{array}$ & $\begin{array}{c}50 \\
(100)\end{array}$ & $\begin{array}{c}50 \\
(100)\end{array}$ & $\begin{array}{c}50 \\
(100)\end{array}$ & $\begin{array}{c}50 \\
(100)\end{array}$ & $\begin{array}{c}320 \\
(\mathbf{1 0 0})\end{array}$ & $\begin{array}{c}100 \\
(100)\end{array}$ & $\begin{array}{c}100 \\
(100)\end{array}$ \\
\hline False & - & - & - & - & - & - & - & - & - & - & - & - & - & - & - \\
\hline Not sure & - & - & - & - & - & - & - & - & - & - & - & - & - & - & - \\
\hline
\end{tabular}

Table.4 A female can't get pregnant during periods

\begin{tabular}{|c|c|c|c|c|c|c|c|c|c|c|c|c|c|c|c|}
\hline \multirow[t]{2}{*}{ Response } & \multicolumn{2}{|c|}{ Purig } & \multicolumn{2}{|c|}{ Balti } & \multirow{2}{*}{$\begin{array}{c}\begin{array}{c}\text { Early } \\
\text { Ado. }\end{array} \\
14-16 \\
\text { Years } \\
\text { N=164 }\end{array}$} & \multirow{2}{*}{$\begin{array}{c}\begin{array}{c}\text { Late } \\
\text { Ado }\end{array} \\
16-18 \\
\text { Years } \\
\mathrm{N}=15 \\
6\end{array}$} & \multicolumn{2}{|c|}{ Girls } & \multicolumn{2}{|c|}{ Mothers } & \multicolumn{2}{|c|}{$\begin{array}{c}\text { Grandmother } \\
\text { s }\end{array}$} & \multicolumn{3}{|c|}{ Females } \\
\hline & $\begin{array}{c}14- \\
16 \\
\text { Year } \\
s \\
N=77\end{array}$ & $\begin{array}{c}16- \\
18 \\
\text { Year } \\
\text { s } \\
\mathbf{N}=83\end{array}$ & $\begin{array}{c}14- \\
16 \\
\text { Year } \\
s \\
N=87\end{array}$ & $\begin{array}{c}16- \\
18 \\
\text { Yea } \\
\text { rs } \\
N=7 \\
3\end{array}$ & & & $\begin{array}{c}\text { Purig } \\
\mathrm{N}=16 \\
0\end{array}$ & $\begin{array}{c}\text { Balti } \\
\mathrm{N}=16 \\
0\end{array}$ & $\begin{array}{c}\text { Puri } \\
\mathbf{g} \\
\mathbf{N}=\mathbf{5 0}\end{array}$ & $\begin{array}{c}\text { Bal } \\
\text { ti } \\
\mathrm{N}= \\
\mathbf{5 0}\end{array}$ & $\underset{\mathbf{N}=\mathbf{5 0}}{\mathbf{g u r i}}$ & $\begin{array}{r}\text { Balti } \\
N=50\end{array}$ & $\begin{array}{c}\text { Girls } \\
\mathbf{N}=32 \\
0\end{array}$ & $\begin{array}{c}\text { Mothe } \\
\mathbf{r s} \\
\mathrm{N}=100\end{array}$ & $\begin{array}{c}\text { Grand } \\
\text { mothe } \\
\text { rs } \\
\mathrm{N}=100\end{array}$ \\
\hline True & $\begin{array}{c}4 \\
(5.1)\end{array}$ & $\begin{array}{c}4 \\
(5.1)\end{array}$ & - & - & $\begin{array}{c}4 \\
(2.4)\end{array}$ & $\begin{array}{c}4 \\
(2.5)\end{array}$ & $\begin{array}{c}8 \\
(5)\end{array}$ & - & $\begin{array}{c}11 \\
(22)\end{array}$ & - & - & - & $\begin{array}{c}8 \\
(2.5)\end{array}$ & $\begin{array}{c}11 \\
(11)\end{array}$ & - \\
\hline False & $\begin{array}{c}4 \\
(5.1)\end{array}$ & $\begin{array}{c}24 \\
(31.1\end{array}$ & $\begin{array}{c}1 \\
(1.1)\end{array}$ & $\begin{array}{c}7 \\
(9.5)\end{array}$ & $\begin{array}{c}1 \\
(1.5)\end{array}$ & $\begin{array}{c}7 \\
(4.4)\end{array}$ & $\begin{array}{c}28 \\
(17.5)\end{array}$ & $\begin{array}{c}8 \\
(5)\end{array}$ & $\begin{array}{c}16 \\
(32)\end{array}$ & $\begin{array}{c}\mathbf{5 0} \\
(\mathbf{1 0} \\
\mathbf{0})\end{array}$ & $\begin{array}{c}50 \\
(100)\end{array}$ & $\begin{array}{c}50 \\
(100)\end{array}$ & $\begin{array}{c}36 \\
(11.2 \\
)\end{array}$ & $\begin{array}{c}66 \\
(66)\end{array}$ & $\begin{array}{c}100 \\
(100)\end{array}$ \\
\hline \multirow[t]{2}{*}{ Not sure } & $\begin{array}{c}73 \\
(94.8 \\
\quad)\end{array}$ & $\begin{array}{c}51 \\
(61.4 \\
)\end{array}$ & $\begin{array}{c}86 \\
(98.8 \\
)\end{array}$ & $\begin{array}{c}66 \\
(90 . \\
4)\end{array}$ & $\begin{array}{c}159 \\
(96.9)\end{array}$ & $\begin{array}{c}117 \\
(75.0)\end{array}$ & $\begin{array}{c}124 \\
(77.5)\end{array}$ & $\begin{array}{l}152 \\
(95)\end{array}$ & $\begin{array}{c}23 \\
(46)\end{array}$ & - & - & - & $\begin{array}{c}276 \\
(86.2 \\
)\end{array}$ & $\begin{array}{c}23 \\
(23)\end{array}$ & - \\
\hline & \multicolumn{6}{|c|}{$\begin{array}{l}\text { Chi-sq between early and late adolescent girls } \\
6.55^{*}, \mathrm{P}=0.03, \mathrm{df}=2\end{array}$} & \multicolumn{2}{|c|}{$\begin{array}{c}\text { Chi-sq between } \\
\text { Purig and Balti } \\
\text { girls } 18.7 * * \\
\mathrm{P}=0.000, \mathrm{df}=2\end{array}$} & \multicolumn{2}{|c|}{$\begin{array}{c}\text { Chi-sq } \\
\text { between } \\
\text { Purig and } \\
\text { Balti mothers } \\
46.6^{* *}, \\
\mathrm{p}=0.00, \mathrm{df}=2\end{array}$} & & & \multicolumn{3}{|c|}{$\begin{array}{l}\text { Chi-sq across generation } \\
=313.5^{* *}, \mathrm{p}=0.000, \mathrm{df}=4\end{array}$} \\
\hline
\end{tabular}

Table.5 Early Menstruation means Early Menopause

\begin{tabular}{|c|c|c|c|c|c|c|c|c|c|c|c|c|c|c|c|}
\hline \multirow[t]{2}{*}{ Categories } & \multicolumn{2}{|c|}{ Purig } & \multicolumn{2}{|c|}{ Balti } & \multirow{2}{*}{$\begin{array}{c}\text { Early } \\
\text { Ado. } \\
14-16 \\
\text { Years } \\
\text { N=164 }\end{array}$} & \multirow{2}{*}{$\begin{array}{c}\text { Late } \\
\text { Ado } \\
16-18 \\
\text { Years } \\
\text { N=156 }\end{array}$} & \multicolumn{2}{|c|}{ Girls } & \multicolumn{2}{|c|}{ Mothers } & \multicolumn{2}{|c|}{ Grandmothers } & \multicolumn{3}{|c|}{ Females } \\
\hline & $\begin{array}{l}14-16 \\
\text { Years } \\
N=77\end{array}$ & $\begin{array}{l}16-18 \\
\text { Years } \\
\mathrm{N}=83\end{array}$ & $\begin{array}{l}14-16 \\
\text { Years } \\
N=87\end{array}$ & $\begin{array}{l}16-18 \\
\text { Years } \\
N=73\end{array}$ & & & $\begin{array}{l}\text { Purig } \\
\mathrm{N}=160\end{array}$ & $\begin{array}{c}\text { Balti } \\
\mathrm{N}=160\end{array}$ & $\begin{array}{l}\text { Purig } \\
\mathrm{N}=50\end{array}$ & $\begin{array}{l}\text { Balti } \\
\mathrm{N}=50\end{array}$ & $\begin{array}{l}\text { Purig } \\
\mathrm{N}=50\end{array}$ & $\begin{array}{l}\text { Balti } \\
\mathrm{N}=50\end{array}$ & $\begin{array}{c}\text { Girls } \\
\mathbf{N}=32 \\
0\end{array}$ & $\begin{array}{l}\text { Mothers } \\
\mathrm{N}=\mathbf{1 0 0}\end{array}$ & $\begin{array}{c}\text { Grand } \\
\text { mothers } \\
\mathrm{N}=100\end{array}$ \\
\hline True & $\begin{array}{c}2 \\
(2.5)\end{array}$ & $\begin{array}{c}8 \\
(\mathbf{1 0 . 3})\end{array}$ & $\begin{array}{c}12 \\
(13.7)\end{array}$ & $\begin{array}{c}39 \\
(53.4)\end{array}$ & $\begin{array}{c}14 \\
(8.5)\end{array}$ & $\begin{array}{c}47 \\
(30.1)\end{array}$ & $\begin{array}{c}10 \\
(6.2)\end{array}$ & $\begin{array}{c}51 \\
(31.8)\end{array}$ & $\begin{array}{c}46 \\
(92)\end{array}$ & $\begin{array}{c}50 \\
(100)\end{array}$ & $\begin{array}{c}50 \\
(100)\end{array}$ & $\begin{array}{c}50 \\
(100)\end{array}$ & $\begin{array}{c}61 \\
(19)\end{array}$ & $\begin{array}{c}96 \\
(96)\end{array}$ & $\begin{array}{c}100 \\
(100)\end{array}$ \\
\hline False & - & $\begin{array}{c}20 \\
(24.0)\end{array}$ & $\begin{array}{c}1 \\
(1.1)\end{array}$ & $\begin{array}{c}14 \\
(19.1)\end{array}$ & $\begin{array}{c}1 \\
(1.1)\end{array}$ & $\begin{array}{c}34 \\
(21.7)\end{array}$ & $\begin{array}{c}20 \\
(12.5)\end{array}$ & $\begin{array}{c}15 \\
(9.3)\end{array}$ & $\begin{array}{c}4 \\
(8)\end{array}$ & - & - & - & $\begin{array}{c}35 \\
(10.9)\end{array}$ & $\begin{array}{c}4 \\
(4)\end{array}$ & - \\
\hline \multirow[t]{2}{*}{ Not sure } & $\begin{array}{c}75 \\
(97.4)\end{array}$ & $\begin{array}{c}55 \\
(71.4)\end{array}$ & $\begin{array}{c}74 \\
(85.0)\end{array}$ & $\begin{array}{c}20 \\
(27.3)\end{array}$ & $\begin{array}{c}149 \\
(90.8)\end{array}$ & $\begin{array}{c}75 \\
(48)\end{array}$ & $\begin{array}{c}130 \\
(81.2)\end{array}$ & $\begin{array}{c}94 \\
(58.7)\end{array}$ & - & - & - & - & $\begin{array}{l}224 \\
(70)\end{array}$ & - & - \\
\hline & \multicolumn{6}{|c|}{$\begin{array}{l}\text { Chi-sq between early and late adolescent girls } \\
51.36^{* *}, \mathrm{P}=0.000, \mathrm{df}=2\end{array}$} & \multicolumn{2}{|c|}{$\begin{array}{l}\text { Chi-sq between } \\
\text { Purig and Balti } \\
\text { girls } 20.4 * * \\
\mathrm{P}=0.000, \mathrm{df}=2\end{array}$} & \multicolumn{2}{|c|}{$\begin{array}{c}\text { Chi-sq } \\
\text { between } \\
\text { Purig and } \\
\text { Balti } \\
\text { mothers } \\
29.0, \mathrm{p}=7 \mathrm{e}- \\
8, \mathrm{df}=1\end{array}$} & & & \multicolumn{3}{|c|}{$\begin{array}{l}\text { Chi-sq across generations } \\
=43.9 * * \mathrm{p}=0.000, \mathrm{df}=4\end{array}$} \\
\hline
\end{tabular}


Fig.1 "Menstruation symbolizes psychological and physical maturity among females"

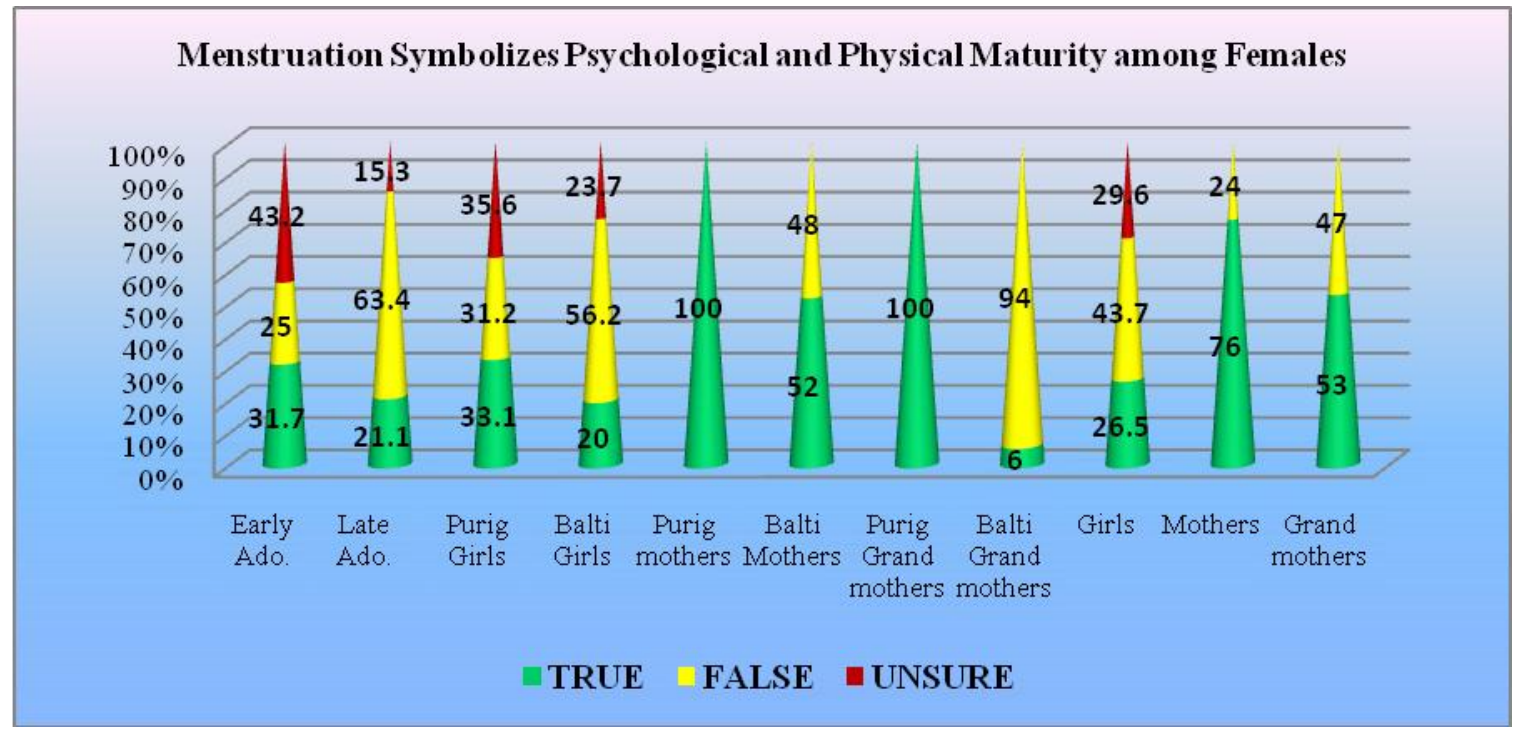

Myth: "It is very Disgraceful for Females when a Man finds out that a Woman is having her Period"

Fact: Menstruation is natural physiological changes in women, so there is no need to feel ashamed in front of males.

Perusal of the data contained in table 3 depict that all $(100 \%)$ respondents across the three generation from both the tribes agreed that it is very disgraceful for females when a man finds out that a woman is having her period. Few women explicitly said that their daughter had been told to maintain silence during their periods. In fact, they were told to pretend in front of male family members to be observing fast during Ramazan month if menstruating but in reality they were not fasting. The data also revealed that girls of Kargil were socialized in such a way that menstruation is a hidden issue and not to be openly discussed especially with males. All the respondents hence thought it is very embarrassing when men finds out that a woman is having her period whether by some accidental situation, like spoiling of dress, avoiding of going to mosque or praying religious prayers etc. In all these situations the other females of the family help to hide the matter from others.

Like many other tribes and communities of India where mention of the topic has been a taboo, here also in Kargil even today menstruation is considered a taboo.

\section{Myth: "A female can't get pregnant during periods"}

Fact: It's not likely, but there's always a chance. Ovulation can be unpredictable and so can menstrual cycles.

Even though sex was prohibited during periods, yet some females also reported the existence of a belief that one cannot get pregnant during this time. Table 4 reveals that all (100\%) sample grandmothers and most mothers $(66 \%)$ reported that it is untrue that one can't get pregnant during periods. The fact is that one can conceive during her monthly cycle as well. They further elaborated that according to Islam 'if one conceives during menstruation then that child will be considered as illegal (haram)' as stated in Holy book Quran. It is impermissible for a 
Muslim to have sexual intercourse with his wife before her menstrual period is over and she has performed the important 'Ghusl' at the end of her period. Only $11 \%$ mothers and $2.5 \%$ adolescent girls thought that one cannot conceive during periods. Further, results reveal that generation wise older generation females were more aware about this myth than younger generation. This may be because the older generation females were married and had more sexual experience than the young girls. Statistical analysis shows highly significant difference across three generations. On the basis of tribe, some Purig girls and mothers believed on this myth. All mothers of Balti tribe considered this as untrue. Similarly, girls of Balti tribe mostly were unsure $(95 \%)$ about the authenticity of this myth.

\section{Myth: "Early Menstruation means Early Menopause"}

\section{Fact: There is no evidence of it and medical science does not approve of this.}

Table 5 clearly shows that Another belief/myth that Early Menstruation means Early Menopause was agreed upon by $100 \%$ grandmothers, $96 \%$ mothers but only $19 \%$ adolescent girls. Some of the older respondents reported that those girls who got their menarche at an early age had maximum chance of attaining menopause at early age but all this is mainly dependent upon individual heredity, which may be from mother's or father's family. Among the three generation more grandmothers and mothers believed on this myth but majority $(70 \%)$ adolescent girls were uncertain about this myth. Comparatively more late adolescent girls believed it as true where as more of early adolescent girls were uncertain about this myth. Irrespective of their tribal affiliation all grandmothers and mothers from both the tribes believed in the concept of 'Early
Menstruation leading to Early Menopause'. However, more Purig tribe girls were unsure of this belief. Statistically significant differences were found among all three generation and also between the two tribes for all the three selected age groups.

The tribal (Purig and Balti) females of Kargil tend to have specific menstrual beliefs, myths, taboos and restrictions. This construction is a part of their unique cultural and ethnic identity and has been passed from generation to generation. Both the tribes conceptualised menstruation as impure, dirty and distasteful phenomenon, which requires no mention in front of others. The elder females especially the grandmothers were very particular about following the menstrual beliefs and taboos. Mothers also to a larger extent followed these concepts. However, some changes were noted among the adolescent girls. They have now started feeling that some of the beliefs were erroneous and were not of much utility.

\section{Recommendation}

For ages women themselves tend to have limited and incorrect information about their own bodies and especially their reproductive system. Females continue to look at their bodies and its processes through the images created by the dominant males around them. Talking and communication about reproductive system and pubertal changes is strict taboo in most cultures including that of Kargil. As a result a number of myths and restrictions continue to flourish around menarche and menstruation. The only way these myths and restrictions are challenged and overcome is by creating awareness about the reproductive system and one's own bodies. Scientific and medical knowledge about puberty and pubertal changes is essential for all adolescent girls so that there is unconditional accepting it. 


\section{References}

Dasgupta, A., and Sarkar, M. (2008). Menstrual hygiene: How hygienic is the adolescent girl? Indian Journal of Community Medicine, 33(2), 77-80.

George, R. (2014). Blood speaks [Blog Post]. Retrieved from https://mosaicscience.com/story/menstr ual-taboo-periods-shame-women

Jane Dineen, 2019 Fighting Menstruation Myths Keeps Girls in School. https://www.unicefusa.org/stories/fighti ng-menstruation-myths-keeps-girlsschool/34921

Khanna, A., Goyal, R. S., and Bhawsar, R. (2005). Menstrual Practices and Reproductive Problems: A Study of Adolescent Girls in Rajasthan. Journal of Health Management, 7(1), 91-107.

Kieren, D. K. (1992). Redesigning Menstrual Education Programs Using Attitudes toward Menstruation. Canadian Home Economics Journal, 42(2), 57-63.

Kivuvani, M. (2013). An Assessment of Baseline Menstrual Hygiene Practices and School Absenteeism in Western
Uganda. Retrieved May 16, 2015, from https://www.unicef.org>schools>files

Shuttle, P., and Redgrove, P. (2005). The wise wound. London: Marian Bayars.

Singh, A. J. (2006). The place of menstruation in the reproductive lives of women of rural north India. Indian Journal of Community Medicine, 31(1), 10-14.

SOS Childrens Village. (2014). Social taboos damage the health of girls and women. Retrieved August 5, 2014, from http://www.soschildrensvillages.org.uk/ news/blog/social-taboos-damage-thehealth-of-girls-and-women.

Ten, V. T. A. (2007). Menstrual Hygiene: A Neglected Condition for the Achievement of several Millennium Development Goals. Europe External Policy Advisors Report, 8(6), 30-42.

Yasmin, S., Manna, N., Mallik, S., Ahmed, A. and Paria, B. (2013). Menstrual hygiene among adolescent school students: An in-depth cross-sectional study in an urban community of West Bengal, India. Journal of Dental and Medical Sciences, 5 (6), 22-26.

\section{How to cite this article:}

Sabiya Asmat, Sarika Manhas and Tashi Dolkar. 2019. Myths Associated with Menarche and Menstruation among Tribal Females of Kargil District, Jammu and Kashmir. Int.J.Curr.Microbiol.App.Sci. 8(06): 2408-2415. doi: https://doi.org/10.20546/ijcmas.2019.806.287 